Revista Facultad 2019

de Ciencias Vol. 27(1)

Económicas enero-junio 2019

ISSN: 0121-6805 · ISSN-e: 1909-7719

- pp. $141-150$

DOI: https://doi.org/10.18359/rfce.3202

\title{
Perfil de responsabilidad social empresarial del sector solidario en Ibagué, Colombia*
}

\author{
Alba Ruth Vargas Montealegre ${ }^{\mathbf{a}}$ - Daniel Augusto León Moreno ${ }^{\mathrm{b}}$
}

\begin{abstract}
Resumen: El presente artículo de investigación muestra la elaboración del perfil de responsabilidad social empresarial del sector solidario en Ibagué, Colombia, sector de gran importancia debido a su enfoque sin ánimo de lucro y a la aplicación de prácticas enfocadas en el desarrollo integral del ser humano. El enfoque es mixto, emplea el análisis de correspondencia como técnica estadística y la descripción de resultados como complemento cualitativo. La recolección de información se realizó a través de un cuestionario aplicado mediante entrevista a empresas del sector solidario en Ibagué. Los resultados permiten establecer las fortalezas y debilidades del sector en materia de responsabilidad social, el nivel de implementación de actividades socialmente responsables y el compromiso de las empresas solidarias con sus colaboradores, asociados y grupos de interés.
\end{abstract}

Palabras clave: responsabilidad social empresarial, gestión humana, perfil empresarial, economía solidaria

Fecha de recibido: 13 de diciembre de 2017 Fecha de aprobado: 15 de noviembre de 2018

Cómo citar: Vargas Montealegre, A. R. y León Moreno, D. A. (2019). Perfil de responsabilidad social empresarial del sector solidario en Ibagué, Colombia. Revista Facultad de Ciencias Económicas, 27(1), 141-150. rev.fac.cienc.econ. doi: https://doi.org/10.18359/rfce.3202

JEL: M14, 015.

* Artículo de investigación. Proyecto Indicadores de responsabilidad social empresarial en medianas y pequeñas empresas de economía solidaria de la ciudad de Ibagué. Entidades financiadoras: Gobernación del Tolima y Universidad de Ibagué. Programa de Cultura Científica. Código. 16-441-SEM. Realizado del 16 de septiembre de 2016 a septiembre de 2017.

a Administradora de empresas y especialista en Gerencia del Talento Humano y Desarrollo Organizacional, Mg Gerencia del Talento Humano, CMg Maestría en Educación. Docente de tiempo completo de la Universidad de Ibagué, tutora del semillero de investigación INFIN, grupo de investigación UNIDERE. Correo electrónico: alba.vargas@unibague.edu.co

b Economista de la Universidad de Ibagué. Se ha desempeñado como asistente de investigación graduado del grupo de investigación UNIDERE, asistente de investigación e integrante del semillero de investigación INFIN. Correo electrónico: leon-9524@hotmail.com 


\title{
Corporate Social Responsibility Profile of the Solidarity Sector in Ibagué, Colombia
}

\begin{abstract}
This research paper discusses the development of the corporate social responsibility profile within the solidarity sector in Ibagué, Colombia. It is a prominent sector because of its non-profit approach and the application of practices focused on the integral development of human beings. The approach hereof is mixed because it uses correspondence analysis as a statistical technique and result description as a qualitative complement. Information was collected through a survey conducted through interviews with companies operating within the solidarity sector in Ibagué. The results identified the strengths and weaknesses of the sector in terms of social responsibility; the implementation level of socially responsible activities; and the commitment of solidarity companies toward their employees, affiliates, and interest groups.
\end{abstract}

Keywords: corporate social responsibility, human management, business profile, solidarity economy

\section{Perfil de Responsabilidade Social Corporativa do Setor Solidário em Ibagué, Colômbia}

Resumo: Este artigo de pesquisa mostra a elaboração do perfil de responsabilidade social empresarial do setor de solidariedade em Ibagué, Colômbia, um setor de grande importância devido à sua abordagem sem fins lucrativos e à aplicação de práticas voltadas para o desenvolvimento integral do ser humano. A abordagem deste instrumento é mista, pois utiliza a análise de correspondência como técnica estatística e a descrição dos resultados como complemento qualitativo. A coleta de informações foi realizada por meio de questionário aplicado por meio de entrevista com empresas operando no setor de solidariedade em Ibagué. Os resultados permitem estabelecer os pontos fortes e fracos do setor em termos de responsabilidade social, o nível de implementação de atividades socialmente responsáveis e o compromisso de empresas solidárias com seus colaboradores, associados e grupos de interesse.

Palavras-chave: responsabilidade social corporativa, gestão humana, perfil empresarial, economia solidária 


\section{Introducción}

En la actualidad, la responsabilidad social empresarial (en adelante RSE) es definida como el conjunto de procesos y actividades coordinadas que desarrollan las organizaciones, desde su modelo de gestión administrativa, con el fin de mitigar el impacto social y ambiental que sus operaciones ocasionan en el entorno. Este concepto establece la RSE como una iniciativa que integra diversas dimensiones de la organización; entre ellas están la gestión ambiental, la gestión humana, las relaciones públicas y la administración financiera, comprendiendo, además, que el compromiso de responsabilidad social con los grupos de interés va más allá de las actividades filantrópicas y las donaciones caritativas (Tello Castrillón y Rodríguez Córdoba, 2016), donde las acciones hacen parte de una gestión integral que permite gestionar la RSE con las causas sociales, económicas y ambientales, con la ética, con la transparencia y en diálogo constante con las partes interesadas o stakeholders (Niño Benavides y Cortés Cortés, 2018, p. 140).

A nivel nacional, se ha demostrado que asociar la RSE con la filantropía ha hecho que el 99,9\% de las empresas en Colombia presenten serias dudas sobre lo que significa ser una organización responsable (Molina, 2009). En vista de ello, y con el propósito de mejorar sus políticas de responsabilidad social, las empresas han optado por orientar sus acciones y programas sociales desde un enfoque estratégico, lo que genera ventajas competitivas que incrementan el valor económico y social de la organización. En este sentido, un programa o proyecto de RSE bien enfocado y con criterios estratégicos definidos puede beneficiar a las empresas, a los grupos de interés y al entorno en el cual operan.

Ahora bien, existe una forma alternativa de hacer economía basada en las personas, el medio ambiente y el desarrollo sostenible llamada economía solidaria, definida según la Ley 454 de 1998 de Colombia como un sistema socioeconómico y cultural caracterizado por emplear prácticas solidarias, democráticas y humanísticas para el desarrollo integral del ser humano. Esta economía se encuentra conformada por organizaciones sin ánimo de lucro, las cuales desarrollan actividades de beneficio social orientadas a la satisfacción de las necesidades de personas, hogares o familias (Manríquez, Martínez y Colín, 2017; Tapia, Tapia, Moscoso y Ortíz, 2017). Sin embargo, el correcto desarrollo de la responsabilidad social en las empresas del sector solidario se ve interrumpido por diferentes factores como la falta de recursos económicos, la poca claridad en la adecuada implementación de acciones socialmente responsables y la necesidad de identificar aquellas actividades destacadas que permitan conocer las fortalezas y debilidades del sector en materia de RSE, lo cual facilitaría la toma de decisiones en las organizaciones relacionadas con sus objetivos, programas e iniciativas sostenibles.

Para abordar la problemática anterior, así como otros temas relacionados con contextos sociales, algunos autores han empleado el análisis de correspondencia como técnica estadística en sus investigaciones. Este es, según Álvarez (2012), una técnica descriptiva multivariante que permite determinar gráficamente la asociación o correspondencia entre categorías de variables, siendo de útil aplicación en trabajos exploratorios donde son pocas e inexistentes las hipótesis previas sobre el comportamiento de la población (Fernández, 2002). Asimismo, este tipo de análisis ha sido utilizado como una herramienta para la caracterización de grupos poblacionales y sectores empresariales, debido a que permite describir y analizar de manera rápida y efectiva las relaciones existentes entre las diversas variables socioeconómicas y demográficas seleccionadas en los estudios (Colmenares, 2017; Jiménez, Mojica, Hernández y Cardona, 2018; Perazzi y Merli, 2017).

Por todo lo anterior, el presente artículo tiene como objetivo establecer el perfil de RSE del sector solidario en Ibagué, a partir de la aplicación de un análisis de correspondencia que permita relacionar las actividades de responsabilidad social con su grado de implementación en las empresas del sector. Además, sintetiza la información proporcionada por 35 empresas del sector solidario en Ibagué, Colombia, sobre la implementación de actividades pertenecientes a las dimensiones de RSE externa: ética, cuidado y conservación del medio ambiente y vinculación con la comunidad; 
así como a las dimensiones de RSE interna: capacitación, salud y seguridad laboral, diálogo social y balance trabajo-familia.

Los resultados del estudio sugieren que las empresas del sector analizado necesitan fortalecer sus conocimientos sobre RSE, debido a la presencia de confusiones en el concepto y los objetivos del tema. Además, pese a que algunas empresas no cuentan con un área exclusiva para el desarrollo de la RSE, las organizaciones solidarias realizan actividades socialmente responsables enfocadas en sus distintos grupos de interés. Específicamente, se destaca la implementación de acciones éticas, sociales y ambientales, así como la aplicación de prácticas de gestión humana relacionadas con la capacitación de personal, la salud y seguridad en el trabajo, el diálogo social y el balance trabajo-familia. De esta manera, se establece que el perfil de RSE del sector solidario en Ibagué se encuentra definido por una serie de actividades implementadas de manera simultánea, correspondientes a todas las dimensiones de la responsabilidad social y a las prácticas de gestión humana más importantes. Lo anterior, en sintonía con Foss, Pedersen, Reinholt \& Stea (2014), quienes afirman que las prácticas laborales a menudo se complementan unas con otras $y$, por tanto, su inclusión solo es efectiva cuando son implementadas en combinación con más prácticas.

Además de esta sección, el artículo se estructura de la siguiente manera: en la sección 2 se plantean las bases teóricas empleadas para el desarrollo de la investigación; en la sección 3 se describe la metodología, las variables y el análisis estadístico empleado; en la sección 4 se muestran los resultados obtenidos. Por último, en la sección 5 se presentan las principales conclusiones sobre la implementación de actividades socialmente responsables y la configuración del perfil de RSE.

\section{Marco teórico}

\section{Perfil empresarial de responsabilidad social}

En respuesta a la necesidad de determinar las actividades socialmente responsables que destacan por su implementación en las empresas, diversos autores han elaborado perfiles de RSE para diferentes regiones y sectores económicos. A partir del análisis cualitativo y cuantitativo de los datos, estas investigaciones identificaron las fortalezas y debilidades de las organizaciones en materia de responsabilidad social, y de esta manera facilitar la toma de decisiones para el mejoramiento de la gestión e implementación de iniciativas responsables.

Así, Morales, Galeano y Muñoz (2014) definieron el perfil de RSE del sector salud en Bucaramanga, observando poco interés por parte de las empresas en la aplicación de acciones responsables, debido a la percepción de estas iniciativas como un gasto en lugar de una inversión, situación que impide la ejecución de políticas de RSE que beneficien el desarrollo sostenible de la región. Asimismo, Sepúlveda, Lizcano, Quintero y Díaz (2013) analizaron la responsabilidad social del sector calzado en la misma ciudad del país, resaltando la poca utilización de métodos de producción ecoamigables y, en general, la falta de políticas sostenibles relacionadas con temáticas medioambientales. De igual forma, y como aspecto positivo, la RSE en los sectores mencionados anteriormente es entendida como una manera de fortalecer el compromiso con los colaboradores, además de ser utilizada a modo de estrategia empresarial para conseguir un mayor reconocimiento en su entorno, como organizaciones socialmente responsables.

\section{Economía solidaria en Colombia y responsabilidad social empresarial}

Existe un amplio número de conceptos para clasificar a las organizaciones que, desde su estructura y finalidad, no pueden ser catalogadas como empresas pertenecientes al sector público o privado. Entre las definiciones más comunes destacan los términos sector social, tercer sector y sector solidario (Obando, 2009). No obstante, sin importar la definición que se use, las empresas pertenecientes a este sector deben cumplir con una serie de características, como ser organizaciones sin ánimo de lucro, ejercer una actividad económica enfocada en cubrir las necesidades de sus asociados y de la comunidad en general, y garantizar la igualdad de oportunidades a sus miembros, sin considerar el monto de los aportes hechos. 
En Colombia, las organizaciones de economía solidaria son clasificadas de acuerdo con su estructura empresarial y finalidad en cooperativas, fondos de empleados y asociaciones mutuales. Según la Unidad Administrativa Especial de Organizaciones Solidarias (2018), las cooperativas son empresas creadas para satisfacer las necesidades de sus asociados, quienes son simultáneamente los aportantes y gestores de la empresa. Por su parte, los fondos de empleados son entidades conformadas por personas independientes y trabajadores asalariados, que ofrecen servicios de crédito a bajo costo. Por último, las asociaciones mutuales son organizaciones constituidas para fomentar la ayuda recíproca entre sus miembros, a través de la prestación de servicios que contribuyan al mejoramiento de su calidad de vida.

En términos de RSE, la correcta aplicación de actividades socialmente responsables puede impactar de manera positiva en los distintos aspectos de la gestión empresarial. Por ejemplo, a nivel externo permite mejorar las relaciones de la empresa con sus grupos de interés y el entorno, mientras que a nivel interno fortalece el vínculo existente entre la alta dirección y las distintas áreas de la empresa. De igual manera, la implementación de acciones responsables trae consigo ventajas para la organización, como el posicionamiento de la marca, la atracción de nuevos clientes, y el mejoramiento del clima laboral, la productividad y el compromiso de los trabajadores (De Miguel, 2011).

No obstante, con frecuencia las organizaciones cometen errores al elaborar e implementar actividades de RSE, lo cual suele generar efectos negativos para la empresa y la sociedad. Según Solano (2005), estas fallas pueden catalogarse como errores de concepción, destacando principalmente la falta de claridad en los objetivos de responsabilidad social, el bajo compromiso en los diferentes niveles de la empresa y la errónea consideración de las acciones filantrópicas y el cumplimiento de lo normativo como el íntegro desarrollo de la RSE. En consecuencia, estas fallas afectan de forma considerable la imagen corporativa, la relación con los grupos de interés y las finanzas de la empresa, al emplear de manera inadecuada los recursos destinados a la ejecución de acciones socialmente responsables.

\section{Metodología}

Para la elaboración del perfil de RSE se utilizó una muestra aleatoria de 35 empresas del sector solidario, las cuales se encontraban operando en Ibagué en 2016. La muestra representativa se constituyó mediante técnicas probabilísticas, con un margen de error de $5 \%$, a partir del listado de entidades regionales elaborado por la Superintendencia de la Economía Solidaria. De acuerdo con su actividad económica, 8 de las empresas consideradas en el estudio corresponden a cooperativas y 27 a fondos de empleados.

La recolección de información se realizó a través de un cuestionario aplicado mediante entrevista al directivo a cargo de las acciones de responsabilidad social en cada organización. El cuestionario fue diseñado con base en la norma ISO 26000 y estaba compuesto por 55 ítems que medían, en escala tipo Likert [1-5], el grado de implementación de actividades relacionadas con la RSE. De los ítems mencionados, 30 correspondían a las dimensiones de responsabilidad social externa y 25 a las dimensiones de responsabilidad social interna.

En adición al componente cuantitativo de la entrevista, fue realizada una serie de preguntas cualitativas, las cuales permitieron complementar la documentación de las actividades socialmente responsables implementadas por las empresas, el beneficio percibido en las organizaciones por la aplicación de estas acciones, así como el nivel de compromiso empresarial con los colaboradores y grupos de interés. Además, en el momento de la entrevista se logró observar si la empresa contaba con un área exclusiva para el desarrollo de la RSE y, en caso contrario, identificar el área o profesional a cargo de las acciones responsables en la organización.

Los datos del estudio fueron examinados desde un enfoque mixto con dos fases de análisis. En primer lugar, se realizó un análisis de correspondencia como método cuantitativo para determinar las posibles tendencias en la implementación de actividades socialmente responsables, que permitieran 
definir el perfil de RSE del sector. Luego, el análisis de los datos fue complementado con la información cualitativa recolectada en las entrevistas, además de la descripción de los resultados obtenidos.

De igual manera, fue estimado el promedio de los ítems en cada dimensión, para tener un indicador consolidado del grado de aplicación de las actividades socialmente responsables. Como se observa en la tabla 1, la implementación promedio del sector solidario en cada dimensión de RSE es alta, y es inferior para la dimensión relacionada con el medio ambiente. Adicionalmente, para evaluar la fiabilidad del instrumento de medición, fue calculado el coeficiente alfa de Cronbach para cada una de las dimensiones de RSE. Los valores observados del coeficiente para cada variable sugieren un nivel adecuado de correlación entre los ítems, cuando estos miden el grado de implementación de cada dimensión.

Para la realización del análisis de correspondencia, la implementación promedio de las dimensiones de RSE en cada empresa fue clasificada en tres grupos, de acuerdo con la intensidad de su aplicación en implementación baja (1-3 valor promedio), implementación media (3-4,5 valor promedio) e implementación alta (4,5-5 valor promedio). Lo anterior debido a que el análisis de correspondencia tiene como condición que las variables por analizar sean categóricas y, por tanto, no es posible desarrollar la técnica con variables continuas.

\section{Resultados}

A continuación, se presentan los resultados del análisis de correspondencia empleando las dimensiones de responsabilidad social como variables categóricas y las empresas encuestadas como variable suplementaria; de esta forma, se define el perfil de RSE del sector solidario en Ibagué.

Como se observa en la tabla 2, las dos primeras dimensiones del estudio representan el 84,47\% de la varianza explicada y, de acuerdo con Higgs (1991), no es necesario considerar dimensiones adicionales debido a que ambas suman más del $70 \%$ de la varianza explicada. Asimismo, la dimensión 1 presenta una mayor relación con todas las variables del estudio, al poseer la inercia más alta del análisis.

Tabla 2. Análisis de correspondencia: inercia y porcentaje de varianza explicada por dimensión

\begin{tabular}{c|c|c|c} 
Dimensión & $\begin{array}{c}\text { Inercia } \\
\text { principal* }^{*}\end{array}$ & $\begin{array}{c}\text { Porcentaje } \\
\mathbf{( \% )}\end{array}$ & $\begin{array}{c}\text { Porcentaje } \\
\text { acumulado }\end{array}$ \\
\hline 1 & 0,23 & 72,36 & 72,36 \\
\hline 2 & 0,03 & 12,11 & 84,47 \\
\hline
\end{tabular}

*Inercia total: 0,321.

Nota: las variables categóricas empleadas en el análisis de correspondencia son las dimensiones de RSE mencionadas anteriormente.

Fuente: elaboración propia.

Los estadísticos de las variables utilizadas en el análisis, como la calidad para cada categoría, el porcentaje de inercia, la correlación y la contribución por dimensión, se presentan detalladamente en la tabla 3. De esta forma, la medida de calidad representa la variación de cada categoría que es reproducida por las dos dimensiones consideradas. En el presente estudio, la calidad es adecuada debido a que la mayoría de categorías tienen mediciones mayores al $80 \%$.

Tabla 1. Estadísticos descriptivos y de fiabilidad para cada dimensión de RSE

\begin{tabular}{|c|c|c|c|c|}
\hline Dimensión & Promedio & Desviación estándar & Alfa de Cronbach & Número de ítems \\
\hline Ética & 4,46 & 0,43 & 0,80 & 11 \\
\hline Medio ambiente & 3,65 & 0,77 & 0,90 & 10 \\
\hline Comunidad & 4,12 & 0,73 & 0,94 & 9 \\
\hline Capacitación & 4,38 & 0,54 & 0,80 & 5 \\
\hline Salud & 4,35 & 0,68 & 0,90 & 5 \\
\hline Diálogo social & 4,47 & 0,49 & 0,83 & 9 \\
\hline Balance trabajo-familia & 4,09 & 0,74 & 0,83 & 6 \\
\hline
\end{tabular}

Fuente: elaboración propia. 
Igualmente, el porcentaje de inercia es la proporción de inercia total causada por cada categoría, la cual, según los resultados, es mayor para las categorías bajas de las dimensiones: vinculación con la comunidad, capacitación y desarrollo y salud y seguridad laboral. Por último, las medidas de correlación y contribución están positivamente relacionadas y, a la vez, presentan resultados inversos para cada dimensión. Así, la dimensión 1 está representada en especial por las implementaciones bajas de todas las dimensiones de RSE, mientras que la dimensión 2 se encuentra compuesta

Tabla 3. Análisis de correspondencia: estadísticos para las variables con normalización estándar

\begin{tabular}{|c|c|c|c|c|c|c|}
\hline \multirow[b]{2}{*}{ Variables } & \multicolumn{2}{|c|}{ General } & \multicolumn{2}{|c|}{ Dimensión 1} & \multicolumn{2}{|c|}{ Dimensión 2} \\
\hline & Calidad & $\begin{array}{l}\text { Porcentaje } \\
(\%) \text { inercia }\end{array}$ & Correlación & Contribución & Correlación & Contribución \\
\hline \multicolumn{7}{|c|}{ Ética } \\
\hline Media & 0,83 & 0,03 & 0,58 & 0,02 & 0,25 & 0,07 \\
\hline Alta & 0,83 & 0,03 & 0,58 & 0,02 & 0,25 & 0,06 \\
\hline \multicolumn{7}{|c|}{ Medio ambiente } \\
\hline Baja & 0,98 & 0,05 & 0,97 & 0,07 & 0,01 & 0,00 \\
\hline Media & 0,98 & 0,01 & 0,79 & 0,01 & 0,18 & 0,01 \\
\hline Alta & 0,81 & 0,02 & 0,18 & 0,00 & 0,63 & 0,10 \\
\hline \multicolumn{7}{|c|}{ Comunidad } \\
\hline Baja & 0,86 & 0,19 & 0,84 & 0,22 & 0,01 & 0,02 \\
\hline Media & 0,66 & 0,01 & 0,03 & 0,00 & 0,63 & 0,07 \\
\hline Alta & 0,72 & 0,03 & 0,26 & 0,01 & 0,46 & 0,13 \\
\hline \multicolumn{7}{|c|}{ Diálogo social } \\
\hline Baja & 0,86 & 0,01 & 0,00 & 0,00 & 0,85 & 0,07 \\
\hline Media & 0,84 & 0,04 & 0,78 & 0,04 & 0,56 & 0,02 \\
\hline Alta & 0,85 & 0,02 & 0,71 & 0,02 & 0,13 & 0,02 \\
\hline \multicolumn{7}{|c|}{ Balance trabajo-familia } \\
\hline Baja & 0,93 & 0,09 & 0,93 & 0,12 & 0,00 & 0,00 \\
\hline Media & 0,69 & 0,00 & 0,52 & 0,00 & 0,17 & 0,01 \\
\hline Alta & 0,55 & 0,02 & 0,31 & 0,01 & 0,23 & 0,03 \\
\hline \multicolumn{7}{|c|}{ Capacitación } \\
\hline Baja & 0,86 & 0,19 & 0,84 & 0,22 & 0,01 & 0,02 \\
\hline Media & 0,66 & 0,02 & 0,08 & 0,00 & 0,59 & 0,11 \\
\hline Alta & 0,65 & 0,02 & 0,13 & 0,00 & 0,52 & 0,09 \\
\hline \multicolumn{7}{|c|}{ Salud } \\
\hline Baja & 0,86 & 0,19 & 0,84 & 0,22 & 0,01 & 0,02 \\
\hline Media & 0,81 & 0,01 & 0,05 & 0,00 & 0,76 & 0,08 \\
\hline Alta & 0,84 & 0,01 & 0,35 & 0,01 & 0,49 & 0,05 \\
\hline
\end{tabular}

Nota: las categorías de las variables corresponden a la clasificación del grado de implementación promedio para cada dimensión en implementación baja, implementación media e implementación alta.

Fuente: elaboración propia. 
mayoritariamente por las implementaciones altas de esas mismas dimensiones.

A partir de la información anterior, la representación gráfica bidimensional es adecuada para la investigación, debido a que ambas dimensiones constituyen la mayor parte de la varianza explicada. La figura 1 muestra los resultados del análisis para las dimensiones de responsabilidad social externa y la posición de las empresas encuestadas en el estudio. La dispersión de las dimensiones y empresas permite identificar una importante relación en la implementación media y alta de las dimensiones: ética, cuidado del medio ambiente y vinculación con la comunidad, las cuales son aplicadas de manera simultánea, y en igual intensidad, por la mayoría de organizaciones solidarias. En contraste, la implementación baja de las actividades comunitarias y medioambientales está relacionada principalmente con una misma empresa del sector, situación que no representa el perfil del sector solidario en materia de responsabilidad social externa.

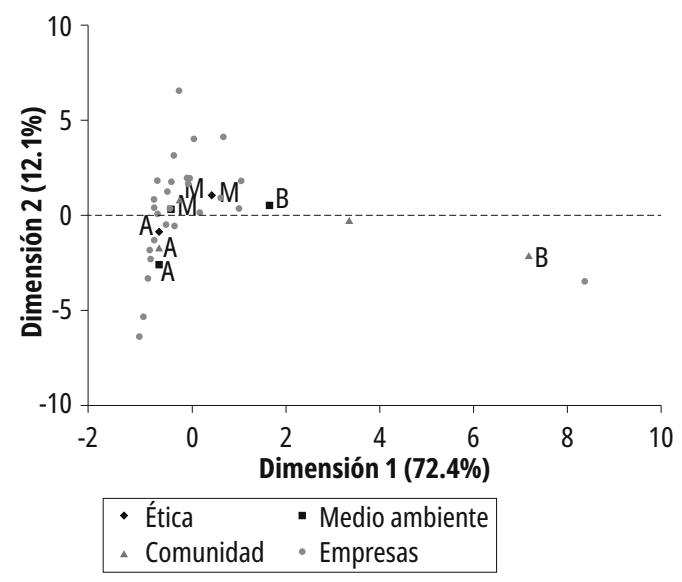

Figura 1. Análisis de correspondencia: representación gráfica de la implementación de las dimensiones de RSE externa del sector solidario en Ibagué

Nota: las categorías empleadas en el análisis se encuentran abreviadas, según el grado de implementación en: B: implementación baja, M: implementación media y A: implementación alta.

Fuente: elaboración propia.

De igual manera, la figura 2 evidencia los resultados del análisis para las dimensiones de responsabilidad social interna y la posición de las empresas solidarias consideradas en la investigación. La dispersión de las dimensiones y empresas permite identificar una fuerte relación en la implementación media y alta de las prácticas de gestión humana: balance trabajo-familia, diálogo social, salud y seguridad laboral, y capacitación y desarrollo; prácticas que son aplicadas de manera simultánea, y en igual intensidad, por la mayoría de organizaciones solidarias. De forma opuesta, la implementación baja de la práctica diálogo social está relacionada de manera individual con algunas empresas del sector, mientras que la implementación baja de las prácticas balance trabajo-familia, salud y seguridad laboral y capacitación y desarrollo se encuentra relacionada con una misma empresa, la cual presenta una implementación baja para todas las dimensiones consideradas en el estudio y, por tanto, no representa el perfil del sector solidario en ningún aspecto de la RSE.

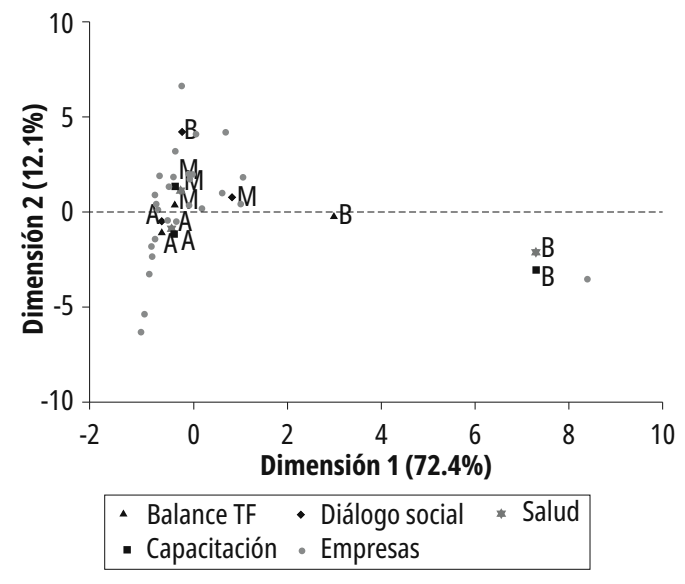

Figura 2. Análisis de correspondencia: representación gráfica de la implementación de las dimensiones de RSE interna del sector solidario en Ibagué

Nota: las categorías empleadas en el análisis se encuentran abreviadas, según el grado de implementación en: B: implementación baja, M: implementación media, y A: implementación alta.

Fuente: elaboración propia.

A partir de los anteriores resultados, es posible identificar que el perfil de RSE de las empresas del sector solidario tiene como principal característica un notable desarrollo de actividades éticas, sociales y ambientales, que reflejan el compromiso del sector con su responsabilidad social externa. De igual manera, las actividades enfocadas en el 
cumplimiento de la responsabilidad social interna se encuentran orientadas principalmente a mejorar las condiciones personales y laborales de sus colaboradores con la implementación de actividades de capacitación, salud y seguridad en el trabajo, y beneficios como horarios flexibles y permisos para actividades familiares, entre otras prácticas.

Como información cualitativa complementaria, cabe destacar la aplicación de actividades éticas, como la capacitación, elaboración y divulgación de reglamentos éticos, la aplicación de normas y códigos que protegen los derechos de la comunidad y los colaboradores de la empresa, el fomento de la dignidad humana y las revisiones permanentes para la prevención de negocios ilícitos. Estas actividades mencionadas tienen especial relevancia en las empresas solidarias debido a la finalidad del sector, que tiene como objetivo el desarrollo integral del ser humano a través de la aplicación de prácticas solidarias, democráticas y humanísticas. Igualmente, como actividades de salud y seguridad laboral por recalcar en las empresas solidarias, se encuentra la ejecución de pausas activas lideradas por profesionales externos, campañas de salud con controles médicos y toma de exámenes, capacitaciones sobre seguridad en el trabajo, el fomento de estilos de vida saludables y la implementación del sistema de gestión de seguridad y salud, de acuerdo con la normatividad legal.

\section{Conclusiones}

El análisis de correspondencia realizado a partir del grado de implementación de las diferentes dimensiones de la responsabilidad social para cada empresa permitió la elaboración del perfil de RSE para el sector solidario en Ibagué. Mediante este último es posible identificar que las empresas del sector se han beneficiado en gran medida con la aplicación de actividades correspondientes al desarrollo de la responsabilidad social, aun cuando algunas de estas organizaciones desconocían los beneficios de implementar actividades socialmente responsables.

También es posible reconocer que las empresas solidarias de la ciudad llevan a cabo actividades dirigidas a la gestión de su responsabilidad social, buscando cubrir las necesidades de sus asociados y de la comunidad en general. No obstante, existe un número importante de empresas que no consideran necesario el mejoramiento de sus procesos medioambientales, debido a que esta acción no hace parte de su estrategia de negocio, con lo cual se limita únicamente al manejo de residuos exigido por la ley.

En lo correspondiente a la situación actual de la responsabilidad social en las empresas solidarias, un gran número de organizaciones no cuenta con un área exclusiva para el desarrollo de la RSE y, por tanto, las actividades son lideradas por otras áreas de la organización, como la dirección ejecutiva o gerencia general. Además, la mayoría de empresas presentan confusiones sobre lo que significa ser una empresa socialmente responsable, al no relacionar la RSE con el desarrollo de una cultura organizacional comprometida con los trabajadores y el entorno. Sin embargo, existe un interés y compromiso por parte de las empresas del sector solidario en avanzar hacia la correcta aplicación de actividades responsables, especialmente en las acciones relacionadas con el bienestar social y laboral de sus colaboradores, así como en la ejecución de proyectos para la vinculación con la comunidad que permitan alcanzar la fidelización de los clientes y mejorar la imagen corporativa de las empresas en materia de responsabilidad social.

Asimismo, los resultados del análisis de correspondencia hicieron posible determinar que las dimensiones de RSE interna y externa son aplicadas de manera simultánea, y en igual intensidad, por las organizaciones del sector solidario en Ibagué, destacando un grado de implementación medio-alto en todas las dimensiones socialmente responsables, con una aplicación levemente menor para las actividades medioambientales. Lo anterior, en contraste con lo señalado por Morales et al. (2014) y Sepúlveda et al. (2013), quienes observaron poco interés en la aplicación de acciones responsables por parte de las empresas pertenecientes a los sectores calzado y salud en Bucaramanga, debido a la percepción de estas iniciativas como un gasto, en lugar de una inversión. 
Como limitaciones del estudio, es importante considerar que la elaboración del perfil de RSE se realizó de acuerdo con la percepción de los directivos en las organizaciones sobre el grado de aplicación de las actividades de responsabilidad social en las empresas solidarias. Por tal razón, como sugerencia para posteriores estudios, se recomienda incluir la percepción de los colaboradores y los distintos grupos de interés sobre la aplicación empresarial de las acciones socialmente responsables. Esto, con el fin de obtener una percepción más amplia, precisa e imparcial del perfil de RSE para el sector objeto de estudio, y de realizar el contraste de los resultados de este estudio con los de otras empresas del sector en otros contextos.

\section{Referencias}

Álvarez, R. (2012). Modelo de evaluación de la calidad para instituciones financieras obtenidas por medio de un análisis de correspondencia y de clúster. Revista Retos, 3(2), 69-94.

Niño Benavides, T. y Cortés Cortés, M. (2018). Comunicación estratégica y responsabilidad social empresarial, escenarios y potencialidades en creación de capital social: una revisión de la literatura. Prisma Social, (22), 127-158.

Colmenares, S. (2017). Análisis multivariante en geografía aplicado al estudio de la pobreza. Terra Nueva Etapa, 33(53), 41-73.

Congreso de Colombia. (1998). "Ley 454 de 1998”, por medio de la cual se regula la economía solidaria en el país. Bogotá, Colombia.

De Miguel, M. (2011). La responsabilidad social empresarial en la pequeña y mediana empresa. Guía de buenas prácticas. Avilés, España: Dirección General de Política de la Pequeña y Mediana Empresa.

Fernández, F. (2002). El uso del análisis de correspondencia simple (ACS) como ayuda en la interpretación del dato en arqueología. Un caso de estudio. Boletín Antropológico, 20(55), 687-713.

Foss, N., Pedersen, T., Reinholt, M. y Stea, D. (2015). Why Complementary HRM Practices Impact Performance: The Case of Rewards, Job Design, and Work Climate in a Knowledge-Sharing Context. Human Resource Management, 54(6), 955-976.
Higgs, N. (1991). Practical and innovative uses of correspondence analysis. Journal of the Royal Statistical Society, 40(2), 183-194.

Jiménez, J., Mojica, J., Hernández, H. y Cardona, D. (2018). Diagnóstico de la innovación y desarrollo tecnológico en el sector hotelero de la región Caribe colombiana. Información Tecnológica, 29(5), 157-164.

Manríquez, N., Martínez, F. y Colín, S. (2017). Reflexiones en torno a la economía solidaria: una revisión de la literatura. Iztapalapa. Revista de ciencias sociales y humanidades, 38(83), 11-42.

Molina, V. (2009). Pymes éticas y responsables. Revista Pesquisa Javeriana, 9, 12-13.

Morales, J., Galeano, C. y Muñoz, J. (2014). Perfil de responsabilidad social empresarial para las entidades promotoras de salud - régimen contributivo de Bucaramanga y su área metropolitana. Revista Cuidarte, 5(1), 633-643.

Obando, D. (2009). Economía solidaria: ¿en función de un desarrollo alternativo o de un neocapitalismo? Alteridad. Revista de Educación, 4(1), 88-97.

Perazzi, J. y Merli, G. (2017). Análisis de la estructura del mercado laboral en Colombia: un estudio por género mediante correspondencias multiples. Cuadernos de Economía, 40(113), 100-114.

Solano, D. (2005). Responsabilidad social corporativa: qué se hace y qué debe hacerse. Journal of Economics, Finance and Administrative Science, 10(18-19), 163-175.

Sepúlveda, J., Lizcano, T., Quintero, I. y Díaz, P. (2013). Perfil de responsabilidad social empresarial del sector de calzado de la ciudad de Bucaramanga. Revista Virtual Universidad Católica del Norte, (39), 212-226.

Tapia, E., Tapia, S., Moscoso, J. y Ortíz, H. (2017). Economía solidaria: estrategia alternativa para el desarrollo local. Visión Gerencial, (2), 313-323.

Tello Castrillón, C. y Rodríguez Córdoba, M. (2016). Fundamentos ético disciplinares de la responsabilidad social organizacional. Revista Venezolana de Gerencia, 21(75), 491-508.

Unidad Administrativa Especial de Organizaciones Solidarias. (2018). Organizaciones economía solidaria. Recuperado de www.orgsolidarias.gov.co/ educación-solidaria/nuestras-organizaciones/organizaciones-economía-solidaria 